\title{
ENT trainee papers presented at the Summer Meeting of ENT Scotland (Scottish Otolaryngological Society), 12 May 2012, Dublane, Scotland, UK
}

\author{
Rhinoplasty: the impact of Scottish government protocols \\ on practice in Scotland
}

P Steele, M Barnes, P Ross

From Ninewells Hospital, Dundee

\section{Introduction}

The Scottish Directorate of Health Workforce and Performance has produced an updated (as of July 2009) Adult Exceptional Aesthetic Referral Protocol. Rhinoplasty is included in this protocol.

\section{Aim}

To investigate the rate of septorhinoplasty and rhinoplasty in Scotland between 2006 and 2010, and the impact of the 2009 protocol.

\section{Methods}

Data on numbers of procedures per specialty, region and year (from 2006 to 2010) were collected from the Information Services Division, Scotland.

\section{Results}

The rate of rhinoplasty performed by ENT and plastic surgeons decreased, while the rate of septorhinoplasty plateaued following introduction of the 2009 protocol. There was significant regional variation in the rates of septorhinoplasty and rhinoplasty, compared with the total population. Overall, the rate at which ENT and plastic surgeons performed septorhinoplasty and rhinoplasty decreased, however the rate for maxillo-facial surgeons increased.

\section{Conclusion}

The protocol has had the intended effect on total septorhinoplasty surgery in Scotland; however, confusion remains as to whom it applies.

\section{Management of human bites to the ear}

A Chin, S Shiekh

From Southern General Hospital, Glasgow

\section{Objectives}

We discuss the difficulties in managing human ear bites. We also highlight two methods, which can be performed by the otorhinolaryngologist, with good cosmetic outcomes.

\section{Methods}

We highlight common difficulties encountered in treating partial avulsion of the ear secondary to human bites. We discuss patient selection, and the technical details involved in achieving good results using the chondrocutaneous advancement flap or rib graft with temporoparietal flap.
Results

We present a pictorial essay of cases using these two techniques, and we discuss patient satisfaction.

\section{Conclusion}

The chondrocutaneous advancement flap, which is often used to reconstruct pinna defects following resection of cutaneous malignancies, is a valuable option for reconstruction of the ear following human bites. Rib graft and temporoparietal flap are useful in the presence of more extensive soft tissue loss.

Development of a screening and splinting service for neonatal ear deformity

L Fraser, N Starritt, L Melia, H Kubba

From the Royal Hospital for Sick Children, Glasgow

\section{Objective}

Splinting neonatal ear deformity within the first few weeks of life has been proven to be successful; however, the opportunity to splint is frequently missed due to lack of awareness amongst healthcare personnel. Our aim was to develop a regional screening service to enable early detection and treatment of such children.

\section{Methods}

We created an information leaflet which was distributed by hearing screeners to all new mothers in Greater Glasgow, shortly after their child's birth, allowing parents to directly refer their baby for assessment if concerned. Babies were reviewed within a week and suitability for splints determined. Parental satisfaction was measured using a questionnaire, and a cost analysis was performed.

Results

Over 18 months, 72 babies were referred, with 44 suitable for splinting. Median age at first review was 13 days, well within the critical period for commencing treatment. Eighty-six per cent of parents rated the efficacy of splints as either excellent or very good, while 95 per cent agreed that they would recommend the service to a friend. Cost analysis revealed a saving of $£ 431$ per child when compared to costs for later corrective surgery.

\section{Conclusion}

Screening and splinting neonatal ear deformity shortly after birth is both acceptable to parents and cost-effective as a service. By detecting treatable children early, we propose that the provision of routine screening and splinting on a wider scale will avoid the psychological burden of ear deformity in childhood, and will also avoid the need for 
later corrective surgery, with significant cost implications for the National Health Service.

Primary otological Wegener's granulomatosis: case series

N Amiraraghi, A Iyer, S Robertson

From NHS Lanarkshire

Objective

Primary otological presentation of Wegener's granulomatosis is very rare. We present four cases of primary otological Wegener's granulomatosis diagnosed by our senior author between December 2010 and December 2011.

\section{Methods}

We present a summary review of the literature regarding Wegener's granulomatosis presenting to ENT specialists. We also present a retrospective case series review of patients presenting to our senior author over the past 12 months with primary otological symptoms of Wegener's granulomatosis.

Results

In 85 per cent of cases, Wegener's granulomatosis presents to ENT services with involvement of the nose or paranasal sinuses. Presentation of primary otological symptoms alone is unusual; such symptoms occur more often (in 38 per cent of cases) as part of a spectrum of presenting symptoms. In our case series, one patient had only serous otitis media which worsened after grommet insertion. Two patients presented with acute ear infection and facial palsy and one with acute mastoiditis. All failed to respond to conventional treatment. All were positive for cytoplasmic antineutrophil cytoplasmic antibody, three had positive histology, and one patient's results were still awaited at the time of writing.

\section{Conclusion}

When acute ear 'infection' fails to respond to conventional treatment, other, rarer causes should be considered. We hope that reporting this series will increase awareness and assist early diagnosis of Wegener's granulomatosis, thus facilitating appropriate treatment and helping prevent systemic involvement.

\section{Ear Popper for eustachian tube dysfunction in adults}

M Shakeel, D Y Veitch

From Aberdeen Royal Infirmary

\section{Background}

Patients with eustachian tube dysfunction have negative pressure in the middle ear as their eustachian tube does not function properly. Most patients experience discomfort and a sensation of fullness in the affected ear. Various treatment options exist for eustachian tube dysfunction, including decongestants, topical nasal steroid preparations, antihistamines, myringotomy with or without insertion of ventilation tube, and insufflation of the eustachian tube.

The Ear Popper (Summit Medical, St Paul, Minnesota, USA) is a modified Politzer device which provides controlled airflow and pressure via a hand-held, battery-operated device. It also enables the air and pressure stream to be timelocked when swallowing.

\section{Methods}

We conducted an observational study assessing the immediate efficacy of the Ear Popper device in patients with eustachian tube dysfunction. Twenty adult volunteers with no history of eustachian tube dysfunction were also invited to use the Ear popper device, as controls. Data collected included demographics, symptomatology, investigations and treatment.

\section{Results}

Seventy-five patients (age range, 20-73 years) were identified over a 2-year period (2010-2011). None were able to auto-inflate their ears using a Valsalva manoeuvre; however, 72 patients were able to do so using the Ear Popper device in the clinic. This provided instant relief from ear discomfort caused by eustachian tube dysfunction. The healthy volunteers also managed to 'pop' their ears with ease using the Ear Popper. No immediate complications were noted in either group.

\section{Conclusion}

Early experience with the Ear Popper device in patients with eustachian tube dysfunction is promising. However, robust studies are needed to objectively confirm the long term efficacy and safety of the Ear Popper device in adults.

\section{Fibrin glue in the management of auricular haematoma}

S Mohamad, M Barnes, S Mahendran, S Jones

From Ninewells Hospital, Dundee

\section{Background}

Auricular haematomas are a common problem requiring emergency ENT management. There is little evidencebased guidance on their optimal management, a relatively high risk of recurrence, and high cosmetic expectations. In recent cases in Dundee, we have formalised a management technique using fibrin glue.

\section{Methods}

The procedure is performed in an out-patient or treatment room setting. After skin preparation and local anaesthetic, an incision is made, the haematoma evacuated, and the cavity washed out with saline. Tisseel fibrin glue is applied diffusely, and a dental roll pressure dressing is applied and secured with a Prolene bead suture. All patients are reviewed after 5 days of oral antibiotics and the suture and dental rolls removed.

\section{Results}

We have managed four cases in this manner: two recurrent and two primary auricular haematomas.

Case 1 was a 32-year-old man with spontaneous right auricular haematoma, who had previously suffered recurrent haematomas after multiple attempts at incision and drainage.

Case 2 was a 17 -year-old man with a 3 -week-old haematoma sustained during trauma while playing rugby.

Case 3 was an 18-year-old man with a recurrent haematoma arising 48 hours after initial aspiration on day three following rugby trauma.

Case 4 was a 16-year-old young man with a 6-day history of haematoma following rugby trauma.

All patients had complete resolution of their haematoma, were satisfied with the cosmetic results, and experienced no complications.

\section{Discussion}

We present four cases of difficult auricular haematoma, two recurrent and two with delayed presentation. All were 
successfully managed with Tisseel fibrin sealant, an inexpensive and easily applied adjunct to out-patient surgical management, the use of which may prevent more invasive and expensive interventions such as suction drainage. Further application may provide evidence of benefit in more routine cases.

\section{Conclusion}

This case series provides evidence that Tisseel fibrin glue is effective in the management of auricular haematoma. A welldesigned, randomised, controlled trial is required in order to reach a conclusive verdict.

Use of fine needle aspiration cytology in a neck lump clinic: three-year review

R Crosbie, E Walker, A Murray, J Dempster

From Crosshouse Hospital, Kilmarnock

\section{Objective}

The aim was to review the results of fine needle aspiration cytology (FNAC) from recent years and to determine whether there were any obvious trends.

\section{Methods}

Retrospective audit of all 'freehand' FNAC procedures performed in Crosshouse Hospital neck lump clinic between May 2008 and August 2011. The correlation between FNAC and histology results was assessed to determine sensitivity and specificity.

\section{Results}

A total of 500 FNAC results were obtained from the 3-year study period. The majority of samples were from lymph nodes and thyroid glands, with the salivary glands, skin and non-specific areas being sampled less frequently. Benign pathology was found for the vast majority of FNAC procedures (77.2 per cent), with much smaller numbers being malignant (12.6 per cent), suspicious of malignancy ( 4.2 per cent) or non-diagnostic ( 6 per cent). Lymph node FNAC produced a higher number of malignant results (30.1 per cent), whereas thyroid and salivary gland FNAC was predominantly benign. A total of 142 histology specimens were reviewed, of which lymph node specimens had the highest rate of malignancy ( 82.4 per cent) compared with the predominately benign disease found in the thyroid (70.4 per cent), salivary gland (77.5 per cent) and skin (100 per cent) samples. Correlation between FNAC and histological results occurred in 89.4 per cent of samples, with lymph nodes being the site least likely to show correlation. The overall sensitivity and specificity of FNAC were 82.4 and 95.9 per cent, respectively. There were no obvious trends in the sites sampled over the three years.

\section{Conclusion}

The sensitivity and specificity of FNAC performed at Crosshouse Hospital neck lump clinic were comparable to those described in the literature. Lymph nodes had a higher prevalence of malignancy on both FNAC and subsequent histological examinations, with thyroid and salivary samples predominately showing benign disease. There were no obvious trends over the three-year study period regarding the site sampled. Comparison with ultrasoundguided FNAC would be a potential next step.
Developing an instant access online emergency logbook for otolaryngology trainees

A Togo, D Duncan

From Western General Hospital, Edinburgh

\section{Objective}

In the past year, otolaryngology trainees have been asked to keep a record of all emergencies managed, with a view to providing this as evidence for the award of the Certification of Completion of Training (CCT). Current methods of recording emergencies are variable and cumbersome, making the process inefficient and often unreliable. We are piloting an otolaryngology emergency log, which is easy to use on any personal computer or portable device with internet connection.

\section{Method}

We have developed a hypertext pre-processor based program which is run from a central server through a web page that has a specific unique resource locator (URL) for each user. Only six data entry lines are required, all driven by 'dropdown' boxes. No patient identifiable data is required. The data are then stored on the server under the user's personal URL, and can be accessed through a link on their web page. The URL can be stored as a bookmark on a portable device, allowing data entry to be undertaken at any time, almost anywhere.

Results

Recording emergencies seen and managed was quick, simple and possible in real time, as was providing a report for use in the Annual Review of Competence Progression (ARCP).

\section{Conclusion}

This is a novel method for collecting the data required of trainees by the ARCP and CCT.

\section{Management of angioedema: the Grampian experience}

S Banik, M Shakeel, B Ram, KW Ah-See

From Aberdeen Royal Infirmary

\section{Introduction}

Angioedema can present with isolated, acute tongue swelling. Most patients respond to standard treatment, but a few do not and may require definite airway support in the form of tracheostomy insertion. This study aimed to determine the most likely cause of acute tongue swelling in our patients, and to identify the predictors of tracheostomy in this cohort.

\section{Patients and method}

We conducted a retrospective audit of the prospectively maintained departmental database, identifying patients who had presented with an acutely swollen tongue over the last two years (2010-2011). Information was collected on demographics, history, examination, investigations and management. Microsoft Excel was used for data collection and analysis.

\section{Results}

A total of 20 patients (13 males and 7 females) presented to the accident and emergency department with an acutely swollen tongue. Four patients presented to us more than once during the study period. Stridor was present in three patients only. Most of our patients had been taking an angiotensin-converting enzyme inhibitor for treatment of hypertension. 
Most patients responded well to adrenaline, steroids and antihistamine treatment. However, two patients required a 'crash' tracheostomy. Routine blood tests were conducted, as well as immunological tests including measurement of complement 3 and 4 levels.

\section{Discussion}

The most common cause of acute tongue swelling in our patients was the use of an angiotensin-converting enzyme inhibitor. Patients failing to respond to adrenaline may require tracheostomy, and a multidisciplinary team approach is recommended when dealing with these patients.

Raised pre-operative serum alkaline phosphatase as a predictive indicator of post-operative hypocalcaemia in patients undergoing total thyroidectomy for Grave's disease

M S Miah, S Mahendran, C Mak, D Smith, G Leese

From Ninewells Hospital and University of Dundee Medical School, Dundee

\section{Background}

Up to 40 per cent of patients suffer post-operative hypocalcaemia after total thyroidectomy. In Grave's disease patients, persistently elevated thyroid hormones are thought to increase osteoclastic activity (with increased serum alkaline phosphatase levels) and hence promote bone resorption. This process ceases after total thyroidectomy, at which stage bone absorbs calcium from the circulation, leading to post-operative hypocalcaemia with normal parathyroid hormone (PTH) levels - the so-called 'hungry bone' syndrome. The aim of this study was to identify whether an elevated serum alkaline phosphatase level is a reliable preoperative predictor of post-operative hypocalcaemia.

\section{Methods}

Data were collected retrospectively from the medical notes and electronic records of patients who had undergone total thyroidectomy between 2003 and 2010. A total of 225 patients were identified. Patients were divided into Grave's and non-Grave's groups. Pre-operative and post-operative biochemical markers were reviewed, including calcium, alkaline phosphatase and PTH. Statistical analysis was conducted using the SPSS (version 15) software program.

\section{Results}

Grave's disease was the most common indication: $n=134$ (60 per cent). Post-operative hypocalcaemia developed in 44 patients (19.5 per cent), and a raised pre-operative serum alkaline phosphatase level was noted in 94 patients (41.8 per cent). A statistically significant association was seen between raised pre-operative alkaline phosphatase levels and post-operative hypocalcaemia, irrespective of PTH levels in Grave's disease: chi-square test, $p=0.037$ (i.e. $p<0.05$ )

\section{Conclusion}

This study demonstrates that a raised pre-operative serum alkaline phosphatase level is a predictive indicator of post-operative hypocalcaemia in total thyroidectomy patients. In addition to meticulous intra-operative techniques, ascertaining the serum alkaline phosphatase level pre-operatively in such patients will help surgeons identify at-risk patients who may benefit from further pre-operative investigations (e.g. vitamin D assay) or use of supplements before surgery.
Sinonasal symptoms in adults with ear disease

M S Miah ${ }^{1,2}$, B-S Yip ${ }^{1}$, M Hussain ${ }^{1,2}$

From ${ }^{1}$ University of Dundee Medical School and ${ }^{2}$ Ninewells Hospital, Dundee

\section{Background}

The association between eustachian tube dysfunction and middle-ear effusion is well established. Studies have also demonstrated pathological changes affecting the middle-ear mucosa, associated with chronic sinonasal inflammation. No previous studies have evaluated symptoms related to sinonasal inflammatory disease in different ear diseases. The aim of this study was to assess the presence of sinonasal symptoms in patients with various ear diseases, using the Dundee Rhinogram.

\section{Methods}

Data were collected prospectively between February and October 2011, from a consecutive cohort of new and follow-up adult patients attending the otology clinic. The presence of sinonasal symptoms was graded using the Dundee Rhinogram. Data were stored using an Excel spreadsheet. Statistical analysis was conducted using the SPSS software program (version 15.0). Student's $t$-test was performed to identify any statistically significant association between ear diseases and sinonasal symptoms.

\section{Results}

A total of 164 patients were assessed. Twenty-five patients had external ear disease, 115 had middle-ear disease (49 with mucosal disease and 66 with squamous disease) and 24 had inner-ear disease. There was no statistically significant association between sinonasal symptoms and external, squamous middle-ear or inner-ear diseases. The mean sinonasal symptoms score for mucosal middle-ear disease was 5.94 (range, $0-32 ; p<0.01$ )

\section{Conclusion}

This study demonstrated a statistically significant association between mucosal middle-ear diseases and sinonasal symptoms. Therefore, assessment of sinonasal symptoms is paramount in patients presenting with an ear symptom, as treatment of inflammatory sinonasal disease may become necessary during management of middle-ear mucosal diseases, in order to achieve a better outcome.

\section{Disseminated neck lumps: where do they come from?}

S Lim, J Yeo, O Hilmi, K Mackenzie

From Glasgow Royal Infirmary

\section{Introduction}

The North Glasgow neck lump clinic was set up to assess patients with potential metastatic neck disease. The majority of malignancies originate in the head and neck region; however, it has long been established that metastases from other sites can present as neck lumps. Classical textbooks suggest that the common sources of these metastases are gastric and lung cancer. This study investigated whether this was still the case, and the subsites in which lumps commonly presented.

\section{Aim}

The study aimed to determine the types of malignancy and the subsites of presentation within the neck. 
Methods

A prospective analysis of all patients referred to the North Glasgow neck lump clinic between October 2005 and October 2010 was carried out. Data specific to patients with metastatic neck disease were recorded, along with the site of presentation.

Results

From October 2005 to October 2010, 2906 patients presented to the clinic. A total of 1027 patients were found to have neck lumps. A total of 775 (75 per cent) patients were diagnosed as having reactive or inflammatory nodes. A total of 252 ( 25 per cent) patients had malignant nodes; in this group, 122 patients had lymphoma, 77 had head and neck malignancy, and 12 had an unknown primary.

Thirty-nine (3.8 per cent) patients had disseminated neck disease from a non head and neck primary (excluding lymphoma patients). In this group, 22 (56 per cent) patients had lung cancer, 5 (13 per cent) had prostate cancer and 3 (8 per cent) had urogenital carcinoma. Other pathologies (23 per cent) included breast cancer, melanoma and oesophageal cancer.

In contrast to head and neck carcinoma, the majority of patients presented with level 5 (39 per cent) and level 4 (30 per cent) lymphadenopathy, followed by level 6 (10 per cent), level 3 ( 8 per cent) and level 2 (6 per cent) disease. Only one patient presented with a posterior neck node.

\section{Conclusion}

Disseminated malignancy can present with neck lumps. The commonest primary site is the lung. In this study, gastric malignancy did not present with any frequency, in contradiction to the published literature. The majority of patients presented with level 5 and 4 nodes.

Can we use nasal peak inspiratory flow to identify treatment responders in the nasal clinic?

N K F Koo Ng, D Young, G W McGarry

From Glasgow Royal Infirmary

\section{Background}

Predicting which chronic rhinosinusitis patients have nasal obstruction which is due to reversible mucosal inflammation could prevent unnecessary surgery.

This study aimed to investigate whether a chronic rhinosinusitis patient's change in nasal peak inspiratory flow following maximal decongestion, at first visit, predicts their response to topical steroids, as measured by the Sinonasal Outcome Test 22.

\section{Methods}

We performed a prospective study of 128 consecutive new adult patients presenting with nasal obstruction due to chronic rhinosinusitis at the Glasgow Royal Infirmary, between January 2008 and July 2010. Nasal peak inspiratory flow was assessed and the Sinonasal Outcome Test 22 questionnaire was completed. Following maximal decongestion of the nose, nasal peak inspiratory flow was re-measured and the difference in peak flow calculated (i.e. peak flow post-decongestion minus peak flow pre-decongestion). Topical steroids were used for at least six weeks. The Sinonasal Outcome Test 22 was then completed again and the difference in results calculated (i.e. post-steroid results minus pre-steroid results).
Results

Data were analysed using means and correlation studies (Spearman's rank correlation). No correlation was found between the difference in nasal peak inspiratory flow results and the difference in Sinonasal Outcome Test 22 results, in chronic rhinosinusitis patients with or without nasal polyps.

\section{Conclusion}

The difference in nasal peak inspiratory flow, following maximal decongestion, does not predict response to topical steroids in chronic rhinosinusitis patients.

\section{Reinke's oedema and malignancy: is there a relationship?}

\author{
S Lim, P Sau, L Cooper, A McPhaden, K Mackenzie
}

From Glasgow Royal Infirmary

\section{Introduction}

As part of a study of the management of Reinke's oedema, we assessed the risk of coexistent pre-malignancy and malignancy in patients with classical Reinke's oedema.

\section{Aim}

To determine the prevalence of dysplasia and malignancy in histologically proven Reinke's oedema.

\section{Methods}

A retrospective analysis of all laryngeal biopsies performed in North Glasgow between 2001 and 2010 was carried out using the North Glasgow Pathology Database. Clinical and pathological data from all patients with histologically confirmed Reinke's oedema were recorded, with specific respect to the reporting of dysplasia and malignancy.

\section{Results}

In the 10-year cohort, 2906 laryngeal biopsies were performed. A total of 190 patients (19 males and 171 females) had histologically proven Reinke's oedema. Of this cohort, the pathologist commented on dysplasia in 172 (91 per cent) patients. Of these patients, 13 ( 8 per cent) had mild dysplasia, 2 ( 1 per cent) had moderate dysplasia and 1 ( 0.5 per cent) had severe dysplasia.

There was no malignancy reported in the cohort.

\section{Conclusion}

In this predominantly female, smoking population, the epithelium appeared to differentiate to benign Reinke's oedema rather than malignancy. Patients can be reassured with regard to the very low risk of malignancy in classical Reinke's oedema. The principal indication for surgical intervention should be the patient's desire to improve their voice.

\section{Preservation of the submandibular gland in neck dissection}

S Goldie, M Jacobs, M Shakeel, K W Ah-See, A Hussain, A Chapman

From Aberdeen Royal Infirmary

\section{Introduction}

The first-echelon lymphatic drainage from the oral cavity is to the submental and submandibular lymph nodes. Involvement of the submandibular gland from extension of an oral cavity cancer is very rare. Our aim was to investigate 
the frequency and the nature of involvement (i.e. submandibular gland or lymph nodes) by assessment of the pathology of neck dissection specimens.

\section{Patients and method}

We conducted a retrospective analysis of neck dissection specimens recorded over a period of 10 years (2000-2010). Information was collected on patients' demographics, primary tumour site and stage, type of neck dissection, and specimen histology (particularly involvement of the submandibular gland and level 1 lymphatics). Microsoft Excel was used for data collection and analysis.

\section{Results}

A total of 520 neck dissection procedures were identified; 342 neck dissections were carried out for patients with head and neck squamous cell carcinoma. Specimens from 210 neck dissections ( 25 bilateral) contained contents of the submandibular triangle, i.e. lymph nodes and/or the submandibular gland.

Of the 235 submandibular glands removed, only 6 (2.6 per cent) were directly involved by extension of the adjacent tumour. Eleven submandibular glands were in close proximity to metastatic level 1 lymph nodes. In all these cases, the primary tumour was in the oral cavity.

\section{Discussion}

On the basis of our findings, we believe that preservation of the submandibular gland is justifiable and safe practice. Clearly, we do not advocate preservation of the submandibular gland in the presence of macroscopic disease.

\section{Does smoking make nasal symptoms worse?}

V Visvanathan, G W McGarry

From Glasgow Royal Infirmary

\section{Aims}

This study aimed to quantify the impact of smoking on sinonasal symptoms, and to measure symptom severity in smokers versus non-smokers. Asthmatics and non-asthmatics were used for control and comparison.

\section{Methods}

The study was performed utilising a prospective computer database containing patients' Sinonasal Outcome Test 22 results, clinical diagnosis, and smoking and asthma status.

Results

Study group. In this comparison, smokers were compared with non-smokers (299 patients; 85 per cent non-smokers and 15 per cent smokers). The mean difference between the patient groups was statistically significant $(p=$ 0.0084).

Comparison groups. The following comparisons were also made: (1) smokers versus asthmatic smokers (57 patients: 75 per cent smokers, 25 per cent asthmatic smokers), mean difference not significant ( $p=0.5518)$; (2) smokers versus asthmatic non-smokers (86 patients, equally divided), mean difference not significant $(p=0.7102)$; and (3) nonsmokers versus asthmatics (299 patients; 85 per cent nonsmokers, 15 per cent asthmatics), mean difference significant $(p=0.0293)$.

\section{Conclusion}

Rhinologists' advice on smoking cessation is evidencebased. Smoking is an independent predictive factor for severity of nasal symptoms. Smokers with asthma do not have more severe symptoms compared with smokers without asthma.

\section{Nasal reconstruction following partial rhinectomy and medial maxillectomy}

A Moore, J Montgomery, J McMahon, S Sheikh

From Southern General Hospital, Glasgow

\section{Objective}

To present the reconstruction of a partial rhinectomy and medial maxillectomy deficit using a radial forearm free flap and paramedian forehead flap.

\section{Methods}

A 66-year-old man with moderately differentiated squamous cell carcinoma of the right nasal vestibule elected to undergo surgical excision and reconstruction.

\section{Results}

The staged reconstruction included a radial forearm free flap; bone from the radius was screwed to the nasal bones. The free flap skin was tubed to create the nasal lining. Dorsal and collumellar grafts were fashioned from costal cartilage. The right alar cartilage was recreated from left conchal bowl cartilage. A paramedian forehead flap replaced the skin of the external nose.

\section{Discussion}

Nasal reconstruction is made difficult by the frailty of the nasal tissues and the need to re-establish normal breathing. The osteocartilaginous framework provides support, which is critical to sustain the soft tissue elements.

\section{Conclusion}

A radial forearm free flap provides both bony support and a nasal lining. It can be supplemented by cartilage grafts and a paramedian forehead flap.

\section{Obstructive sleep apnoea related quality of life in} children with Down's syndrome

N Oozeer ${ }^{1}$, J Park ${ }^{1}$, H Kubba ${ }^{2}$

From ${ }^{1}$ Gartnavel General Hospital, and ${ }^{2}$ The Royal Hospital for Sick Children Glasgow

\section{Background}

Individuals with Down's syndrome have a reported incidence of obstructive sleep apnoea syndrome (OSA) of 30-60 per cent. We aimed to determine the OSA-related quality of life $(\mathrm{QoL})$ of children with this condition.

\section{Methods}

A cross-sectional study was carried out involving pre-school children registered with the multidisciplinary Down's syndrome clinic. The parents of 43 patients were asked to complete the OSA-18 questionnaire. A score of $\geq 60$ was considered abnormal. 


\section{Results}

A 70 per cent response rate was obtained. There was no sex preponderance, and age ranged from five months to four years and seven months. The average overall score was 46.7 (range, 19-110). There was no sex difference. The worst scores were seen in three- to four-year-old children (mean, 61.1). Eight children who had undergone adenoidectomy with or without tonsillectomy scored worse compared with a non-intervention group (means, 67.1 versus 39.3, respectively).

\section{Conclusion}

We detected worse OSA-related QoL in children who had undergone surgery (post-operatively); this may reflect the aggressive nature of these patients' airway condition, which led to surgical intervention.

Is paranasal sinus disease more common in patients with chronic otitis media? Comparing Lund-Mackay scores of patients with chronic otitis media versus nasal disease or cochlear implantation

M Yaneza ${ }^{1}$, M Shanks ${ }^{2,3}$, O Hilmi $^{1}$, L Cooke $^{4}$

From ${ }^{1}$ Glasgow Royal Infirmary, ${ }^{2}$ Scottish Cochlear Implant Programme, Kilmarnock, ${ }^{3}$ University Hospital Crosshouse, Kilmarnock, and ${ }^{4}$ Gartnavel General Hospital, Glasgow

\section{Aim}

This study aimed to determine whether paranasal sinus disease is more common in patients with chronic otitis media.

\section{Methods}

Patients who had undergone temporal bone computed tomography (CT) that also included the paranasal sinuses, as part of investigations for cochlear implantation, were used as the control population (as the CT was performed for non-rhinological disease). Patients who had undergone endoscopic sinus surgery or endoscopic polypectomy were used as a comparator population. The Lund-Mackay score was calculated for 60 patients with chronic otitis media, 60 patients from the Scottish Cochlear Implant Programme, and 60 patients who had undergone endoscopic sinus surgery or polypectomy. The scores of the three groups were compared. A Lund-Mackay score of more than 4 was regarded as abnormal and indicative of paranasal sinus disease.

\section{Results}

Thirty-five per cent $(n=21)$ of patients with chronic otitis media had a Lund-Mackay score of greater than 4, compared with 60 per cent $(n=36)$ of the cochlear implant group patients and 91.2 per cent $(n=55)$ of the paranasal sinus disease patients. The mean Lund-Mackay scores in the chronic otitis media, cochlear implant and paranasal sinus disease groups were 4.6, 5.5 and 13, respectively. There was no statistical difference between the chronic otitis media and cochlear implant groups (Mann-Whitney test, $p=0.128)$.

\section{Conclusion}

Patients with chronic otitis media do not have more paranasal sinus disease compared with a normal population. 